\title{
PARTICIPACIÓN DE LOS ALUMNOS DE EDUCACIÓN PRIMARIA EN LOS PROCESOS DE EVALUACIÓN Y CALIFICACIÓN
}

Participation of Primary Educations students in evaluation and qualification processes

Participação dos alunos da Educação Primária nos processos de avaliação e classificação

\author{
Marcos Herranz Sancho (1)
}

(1) Junta Castilla y León, España. Teléfono 659595790. Correo electrónico: marcosancho26@gmail.com

\section{Resumen}

El objeto principal de este trabajo es mostrar los efectos que se consiguen y que se persiguen dotando a los alumnos de participación dentro de sus procesos de evaluación y de calificación. De las distintas formas y modalidades en las que se puede producir esa participación expondremos las que más se adecúan a nuestra forma de entender la práctica educativa. Existen razones pedagógicas y éticas por las que apostamos por la participación de los alumnos en sus procesos de evaluación. Nuestra experiencia nos lleva a afirmar que las consecuencias de su puesta en funcionamiento han sido enormemente positivas para todas las personas implicadas en los procesos de enseñanza y aprendizaje (alumnos y profesores) y para el propio proceso.

Palabras clave: Participación; Evaluación; Ética; Aprendizaje.

\begin{abstract}
The main aim of this work is to show the effects that are being achieved by involving students in their assessment and qualification processes. We will expose those forms and modalities in which such participation can take place that are more suitable for our way of understanding the educational practice. There are pedagogical and ethical reasons why we opt for the participation of students in their evaluation processes. Our experience leads us to say that the consequences of its implementation have been extremely positive for all those involved in the teaching and learning processes (pupils and teachers) and for the process itself.
\end{abstract}

Keywords: Participation; Assessment; Ethics; Learning. 


\section{Resumo}

O objetivo principal de este trabalho é mostrar os efeitos que se alcançam e que se perseguem, quando os alunos participam nos seus processos de avaliação e classificação. Das distintas formas e modos de operacionalizar essa participação, apresentaremos as que mais se adequam à nossa forma de entender a prática educativa. Existem razões pedagógicas e éticas que justificam a nossa aposta na participação dos alunos nos seus processos de avaliação. A nossa experiência leva-nos a afirmar que as consequências desta forma de atuar, foram enormemente positivas para todas as pessoas implicadas nos processos de ensino e aprendizagem (alunos e professores) e para o próprio processo.

Palavras-chave: Participação; Avaliação; Ética; Aprendizagem

\section{1. ¿Cómo participar?}

Los alumnos pueden participar en sus procesos de evaluación y de calificación de muy diversa formas utilizando distintas técnicas e instrumentos.

Por nuestra experiencia pensamos que las principales formas de participación del alumnado en los procesos de evaluación y calificación se dan mediante las técnicas de la autoevaluación, la evaluación compartida, la autocalificación, la calificación dialogada y la coevaluación.

Diversos autores han definido la autoevaluación como la evaluación que una persona realiza sobre sí misma. Nosotros entendemos que es un proceso más complejo ya que la autoevaluación dentro de los procesos educativos ha de ser la apertura de un proceso de reflexión interno serio y sincero. Será realizado por parte de la persona o personas afectadas, ya sean profesores o alumnos, con el fin principal de la autocrítica constructiva y la posible toma de decisiones para conseguir mejoras en los aprendizajes de los alumnos, en las prácticas docentes del profesor y en los propios procesos de evaluación.

La evaluación compartida es el paso lógico y natural que se realiza posteriormente a la autoevaluación del alumno. Se trata de un diálogo entre el profesor y el alumno para tratar aspectos sobre los procesos de aprendizaje y llegar a acuerdos sobre las posibles decisiones (López 2006). El gran aporte de la evaluación compartida es la información que se genera en ambos sentidos entre las personas que la practican, la calidad de dicha 
información y la utilización de la misma. La toma de decisiones compartida favorece enormemente el proceso de evaluación y los posteriores procesos de enseñanza y aprendizaje.

La autocalificación se produce cuando un alumno se pone a sí mismo la calificación que cree que merece de manera razonada y basándose en unos criterios establecidos que normalmente se han determinado previamente (López 2006) entre el profesor y los alumnos (López 2012). La riqueza del proceso se da cuando se pide a los alumnos que abran un proceso de reflexión en torno a su práctica educativa y se compare con respecto al grado de cumplimiento de los criterios establecidos.

La calificación dialogada es un proceso de diálogo que se establece entre el profesor y el alumno para tratar aspectos relacionados sobre la autocalificación previa del alumno en el que el profesor manifiesta su postura sobre la calificación de ese alumno (López 2006). El objetivo será llegar a un acuerdo con respecto a la calificación definitiva basándose en unos criterios de calificación previamente establecidos (López 2012) y será una consecuencia coherente y lógica de la evaluación compartida (López, Pascual y Barba 2005).

Hablamos de coevaluación cuando se produce una evaluación entre iguales; por ejemplo cuando un alumno evalúa a un compañero.

\section{2. ¿Por qué participar?}

La participación de los alumnos en sus procesos de evaluación es un factor que enriquece enormemente dicho proceso para todos los agentes que intervienen en él.

Calatayud (2008) muestra los beneficios de la participación de los alumnos en la evaluación mediante la autoevaluación. Destaca los siguientes:

1. Es uno de los medios para que el alumno tome conciencia de su progreso individual.

2. Ayuda a los alumnos a responsabilizarse de sus actividades.

3. Es un factos clave de motivación hacia el aprendizaje.

4. Ayuda a reflexionar individualmente sobre el proceso de enseñanza y aprendizaje.

5. Puede sustituir a otras estrategias para atender a la diversidad.

6. Ayuda a profundizar en el autoconocimiento y comprensión del proceso realizado.

7. Posibilita la autonomía y la autodirección del alumno. 
Para Herranz (2015) las razones más importantes para dotar a los alumnos de participación en sus procesos de evaluación y calificación tienen que ver con los siguientes factores:

1. Ser consecuentes con nuestra forma de entender la educación.

2. Se trata de una actividad enormemente motivante para los alumnos.

3. Se produce mayor dominio del contenido por la coevaluación y el constante feedback.

4. Se fomentan aprendizajes muy significativos.

5. Nos aporta información para mejorar.

6. Se da responsabilidad al alumno para que sea el principal protagonista de su propio aprendizaje fomentando procesos de reflexión interna buscando la formación de niños responsables y personas responsables en el futuro.

López, Herranz y Mínguez (2018) analizan las ventajas de la participación de los alumnos en los procesos de evaluación una vez recopilados numerosos estudios. Las principales parecen ser:

- Mejora el aprendizaje.

- Mejora la autonomía personal, la autorregulación y los procesos de aprender a aprender.

- Desarrolla la capacidad de análisis crítico y la autocrítica.

- Es un proceso fundamental en la formación de personas responsables y en el desarrollo de una educación democrática.

- Suele ser muy útil en la mejora del clima de aula y en la resolución de problemas de convivencia en el aula y/o en el centro.

- En algunos casos, también es una cuestión de coherencia con las convicciones educativas del profesorado y con los proyectos curriculares propios o del centro.

\section{3. ¿Para qué y para quién proponemos la participación?}

En primer lugar, para intentar conseguir que la evaluación sea una actividad de aprendizaje (Álvarez Méndez 2001) y no de sanción y/o control. Se trata de que las personas implicadas puedan aprender más utilizando los errores como recursos didácticos y como generadores de nuevos conocimientos y aprendizajes. Todo lo contrario sería utilizar esos errores como método de sanción, castigo o reproche. De la misma manera tampoco consideramos adecuado la utilización de la evaluación para 
mantener el control del aula a nivel de respeto de normas de convivencia y/o a nivel disciplinario.

En segundo lugar para poner la evaluación al servicio todos los implicados en la misma, ya sean alumnos, profesores y/o el propio proceso de enseñanza y aprendizaje. De esta manera se podrán beneficiar de ella (Santos Guerra 1993) para obtener nuevos aprendizajes a partir de la información que surge de la evaluación. Al plantearnos estas cuestiones estaríamos considerando una serie de intereses, intenciones, ideologías, valores, expectativas, exigencias y principios (López 2006) determinados. En el análisis de los mismos entrarían las consideraciones éticas y las concepciones educativas personales de cada docente.

\section{Referencias}

Álvarez Méndez, J. M. (2001). Evaluar para conocer, examinar para excluir. Madrid: Morata.

Calatayud Salóm, M. A. (2008). La evaluación como estrategia de aprendizaje para atender a la diversidad.

wwwww.educaweb.com/noticia/2008/01/28/autoevaluacion-como-estrategiaaprendizaje-atender-diversidad-2752/

Herranz Sancho, M. (2015). Resultados de una experiencia de autoevaluación y evaluación compartida en el área de Educación Física en la etapa de Educación Primaria. Revista arbitrada del centro de investigación y estudios gerenciales, $n^{\circ} 21, p p .126-141$.

López Pastor, V. M. (Coord.) (2006). La evaluación en educación Física. Revisión de los modelos tradicionales y planteamiento de una alternativa: la evaluación formativa y compartida. Madrid: Miño y Dávila.

López Pastor, V. M. y Gea Fernández, J. M. (2012). Innovación, discurso y racionalidad en Educación Física. Revisión y prospectiva. Revista Internacional de Medicina y Ciencias de la Actividad Física y el Deporte, no 38, 245-270.

López Pastor, V. M., Herranz Sancho, M. y Mínguez Gómez, P. L. (2018). Evaluación formativa y compartida para una Educación Física crítica. En Lorente Catalán, E. y Martos García, D. (eds.), Educación Física y pedagogía crítica. Propuestas para la transformación personal y social. (pp. 325-348). Lleida y Valencia: Universidad de Lleida y Universidad de Valencia. 
López Pastor, V. M., González Pascual, M. y Barba Martín, J. J. (2005). La participación del alumnado en la evaluación: la autoevaluación, la coevaluación y la evaluación compartida. Revista Tándem, nº 17, 21-37.

Santos Guerra, M. A. (1993). La evaluación: un proceso de diálogo comprensión y mejora. Málaga: Aljibe. 